\title{
Path-Dependent Gaze Control for Obstacle Avoidance in Vision Guided Humanoid Walking
}

\author{
Javier F. Seara, Klaus H. Strobl, and Günther Schmidt \\ Institute of Automatic Control Engineering \\ Technische Universität München, 80290 Munich, Germany \\ Javier.Fernandez.Seara@ei.tum.de
}

\begin{abstract}
This article presents a novel gaze control strategy for obstacle avoidance in the context of vision guided humanoid walking. The generic strategy is based on the maximization of the predicted visual information. For information/uncertainty management a new hybrid formulation of an Extended Kalman Filter is employed. The performance resulting from this view direction control scheme shows the dependence of the intelligent gazing behavior on the preplanned local path.
\end{abstract}

\section{INTRODUCTION}

The achievement of the high level of coordination required between vision and locomotion has become a major topic in current research into humanoid robotics. Most of the groups working in this field, seem to have accepted several antrophomorphic characteristics. These include biped structures and the presence of some kind of active perception system, for example based on stereo vision [1], [2]. Starting with these premises and with the goal of increasing the degree of independence, or rather autonomy, of the robot, some intelligent means of controlling perception become necessary in order to guarantee that the humanoid is provided with the highest possible amount of task-dependent relevant information.

This article is organized as follows: In Section II the problem of gaze control in vision guided walking is defined, and the requirements on the gaze controller are stated. Section III gives an overview of the information management mechanism used. This represents the starting point for the predictive view direction control scheme presented in Section IV. Section V demonstrates with simulations the applicability of this approach. The article presents in Section VI an outlook on possible improvements that can be obtained by the proposed strategy.

\section{PROBLEM DEFINITION}

For a vision guided walking machine to execute a given locomotion task, the guidance system must provide appropriate step sequences which should allow the robot to navigate and reach the goal position taking into account the environmental situation. In case of a vision guided humanoid, the information about the environment is provided by an active vision system. The acquisition and

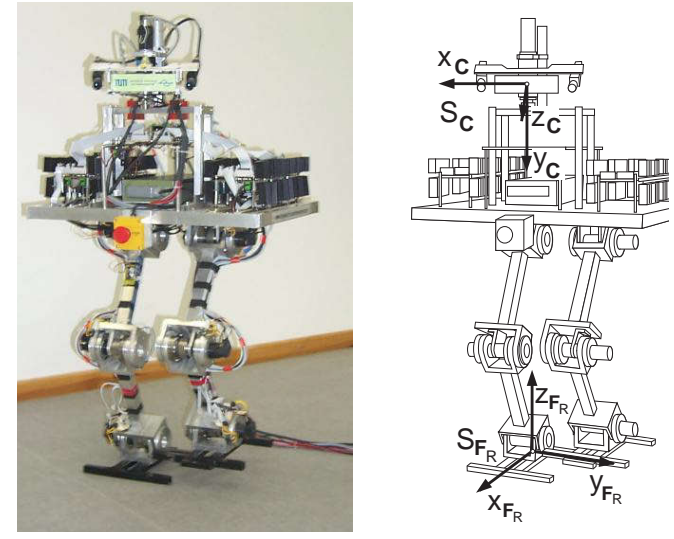

Fig. 1 Biped robot with camera reference frame $S_{C}$ and foot reference frame $S_{F_{R}}$

management of this information can be accomplished by a gaze control scheme selecting the orientation of the sensor, such that the relevant objects of the scene are kept within the sensor's limited field of view. This is also denoted as the "where to look next?"task.

In our research we consider an information management scheme, which registrates and administers the uncertainties associated with the different processes involved in the perception based locomotion [3], [4]. This description of the accumulated information together with the uncertainties in the measurements can provide a basis for the task-oriented gathering of the relevant information. Since uncertainty must be redefined in the context of biped walking, we will refer to it with the notion Incertitude $\nu$. We define Incertitude as a measure of the uncertainty related to the total amount of reliable knowledge required for the successful fulfillment of a given task. This can be interpreted as a task-dependent mapping of the various uncertainties. We define the Information Content of a view situation as a measure of the degree to which perception under these terms is capable of reducing Incertitude.

\section{COUPLED HYBRID EKF FOR INFORMATION MANAGEMENT}

In [3] we presented an information management scheme for active gaze control in the context of vision guided humanoid walking. The proposed biologically inspired 
first approach to gaze control was developed for obstacle avoidance tasks and is based on the maximization of shortrange predicted visual information. The quantification of the information required a stochastic model of both the robot motion and the perception system. In this work, a visual perception model for a stereo-camera pair was considered. Its capability of measuring distances is used, and both the reconstruction formulation and the error representation have been discussed in detail in [4].

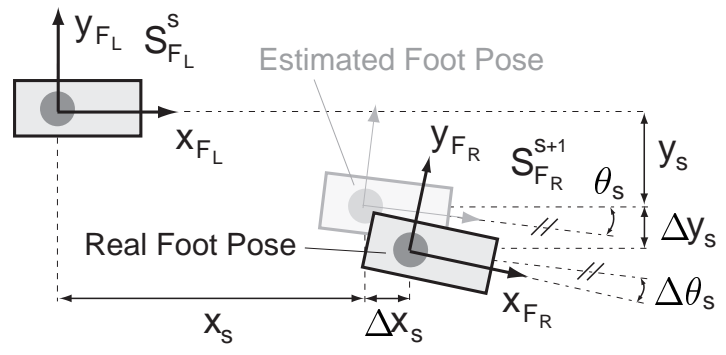

Fig. 2 Step errors of a biped in dead-reckoning.

The biped robot model considered was a simplified general model inspired by real walking machines [5]. The robot model comprises kinematic transformations between different reference coordinate frames. ${ }^{C} \boldsymbol{T}_{F_{R, L}}$ describes the transformation between the camera reference frame $S_{C}$ and the current foot frame $S_{F_{R, L}}$, cf. Fig. 1. This frame changes at the beginning of each step, e.g. $S_{F_{L}}^{s}$ to $S_{F_{R}}^{s+1}$ in Fig. 2, and remains attached to the currently standing foot for the step duration. This reflects the discrete character of the biped locomotion and leads to a modeling of the walking process taking into account the dead-reckoning step errors, see Fig. 2. Both, the relative position of the next foot and its orientation are considered uncertain with errors $\Delta x_{s}, \Delta y_{s}$, and $\Delta \theta_{s}$.

The information/uncertainty management, i.e. relationship between system state estimation and the active measurements, employed a coupled (considering crosscovariances) hybrid (reflecting the discontinuous character of biped walking) Extended (copes with nonlinear systems) Kalman Filter. With the process modeling for walking explained earlier, the state transition function $f$ for the new filter formulation can be represented as follows:

$$
\begin{aligned}
\boldsymbol{x}_{k+1} & =\boldsymbol{f}\left(\boldsymbol{x}_{k}, \boldsymbol{u}_{k+1}, \boldsymbol{w}_{k}\right)= \\
& =\boldsymbol{x}_{k}\left(1-\gamma_{k+1}\right)+\boldsymbol{f}_{s}\left(\boldsymbol{x}_{k}, \boldsymbol{w}_{k}\right) \gamma_{k+1},
\end{aligned}
$$

where $\gamma_{k+1}$ is a binary variable representing the current control vector: $\gamma_{k+1}=0$ indicates no change in the coordinate frame, whereas $\gamma_{k+1}=1$ means an instantaneous change in the coordinate frame. Consequently, the function $\boldsymbol{f}_{s}$ is defined as the transformation of the state vector $\boldsymbol{x}$ when the standing foot changes. On the other hand, an appropriate measurement equation must be chosen based both on the stereo-vision system properties and the object features detected by it, refer to [4] for details.

\section{GAZE CONTROL STRATEGY FOR OBSTACLE AVOIDANCE}

Walking in an environment with obstacles requires a reliable, predictive classification and pose estimation of all obstacles in the walking trail. As discussed in Section II, an active vision system needs a view direction control scheme to determine where to direct the sensor so that the relevant objects in the scene are within its field of view. A simple reactive controller neglecting the delay due to non-zero image acquisition and processing times seems therefore inappropriate for an active vision application, which requires not only a reactive component but also a purposive task-dependent active behavior. Prediction proves to be an appropriate method to improve performance of such a system.

\section{A. Predictive Gaze Control Strategy}

With the concepts of Information Content and Incertitude defined earlier, we present a predictive pathdependent gaze control strategy for an active vision system based on the maximization of the predicted visual information. The approach is as follows: Using the accumulated available information about the scene (for example a 3D-map, i.e. position ${ }_{F} \boldsymbol{x}_{i}$ of object $i$ relative to the current foot frame $S_{F}^{s}$ and its covariance matrix $C_{i j}$ ), the current observer location ${ }_{0} \boldsymbol{x}_{0}$ - which forms together with ${ }_{F} \boldsymbol{x}_{i}$ the state vector $\boldsymbol{x}$, present motion parameters, and the current task (which determines the relationship $\nu_{i}=f\left(\boldsymbol{x}, \boldsymbol{C}_{i j}\right.$, task $\left.)\right)$, the gaze controller predicts the (quasi-)optimal pose of the visual sensor for a future view situation $\boldsymbol{\Omega}_{\star}$ (predicted variables are denoted by - and optimal ones by index $\star$ ). The optimal pose, i.e. position and orientation, but commonly and without loss of generality only orientation (view direction), is found by selecting the state of maximum predicted Information Content, $\widehat{\mathcal{I C}}_{\max }$, among a set of predicted states. This can be expressed by:

$$
\widehat{\boldsymbol{\Omega}}_{\star}=\arg \max _{\widehat{\boldsymbol{\Omega}}} \sum_{i=1}^{N} \widehat{\mathcal{I C}}_{i}\left(\widehat{\boldsymbol{\Omega}},{ }_{0} \boldsymbol{x}_{0},{ }_{F} \boldsymbol{x}_{i}, \boldsymbol{C}_{i j}, \nu_{i}\right),
$$

subject to

$$
\begin{aligned}
& \boldsymbol{\Omega}_{\min } \leq \widehat{\boldsymbol{\Omega}} \leq \boldsymbol{\Omega}_{\max } \text { and } \\
& \boldsymbol{r}(\dot{\boldsymbol{\Omega}})=\mathbf{0},
\end{aligned}
$$

where Eq. (3) are upper and lower bounds for the active vision system parameters and Eq. (4) guarantees a steady gaze behavior without abrupt view direction changes. The latter would disturb the perception process. Eq. (4) takes into consideration the observer's system dynamics and limits the performance (e.g. speed) of saccades.

\section{B. Incertitude Definition: Path-dependence}

Next, a formulation of Incertitude related exclusively to an obstacle avoidance task is presented, see Section II. It is obvious that the existence of obstacles to be avoided depends on which path the biped robot is to follow. 
This fact must be taken into consideration by the gaze controller. Therefore, a definition of Incertitude related to an obstacle avoidance must include the planned local path - assumed to be given - in order to optimize the resulting gaze behavior. The main task considered here is collision avoidance with obstacles present in the environment. This implies that the relevance of an obstacle for the gaze control depends, in this case, on the risk of collision that this object may represent during robot locomotion. In the following some concepts related to this aspect are discussed in more detail.

\section{Critical Points for Collision}

One of the main objectives of safe locomotion is avoiding collisions. Given a path through a scenario with different objects, there exist several points on this path in which the danger of colliding with some obstacle is greater than in others. These points are named critical points for collision and are defined here as the points on the path where the distance $d_{i}$ between the standing foot $S_{F}$ and the $\operatorname{Obj} i$ has a local minimum - taking into account the discrete character of the walking process. These points and their critical distances - when the robot passes by for the $j$-th time at the instant $c_{i j}$, cf. Fig. 3 - can be characterized by the position ${ }_{F} \boldsymbol{x}_{i}^{c_{i j}}$ of the object $i$ relative to the foot frame $S_{F}^{c_{i j}}$ and its covariance matrix $\boldsymbol{C}_{i i}^{c_{i j}}$.

The existence and location of these critical points must be estimated in time, i.e. before the humanoid arrives there, in order to adapt its behavior for coping with them. The estimation of both the position ${ }_{F} \widehat{\boldsymbol{x}}_{i}^{c_{i j}}$ and the position covariance matrix $\widehat{\boldsymbol{C}}_{i i}^{c_{i j}}$ of the object $i$ at the critical instant $c_{i j}$ rests on the propagation of the system state; see the left-hand column in Fig. 4. Estimation can be achieved by linearizing of the system equations, Eq. (1). Information inputs for these estimates are past measurements (in step $s$ and previous).

Incertitude Definition for Obstacle Avoidance

Based on the presented aspects, a definition of obstacle avoidance Incertitude is proposed corresponding with the concept of Probability of Collision. This Incertitude is now $\nu_{i j}^{s}=\widehat{P}_{i}^{c_{i j}}$, i.e. the predicted probability of collision with the object $i$ at $c_{i j}$, where $c_{i j}$ represents a descriptor for a critical point. This gives an idea of the security by passing the object $i$ the $j$-th time. $\nu_{i j}^{s \mid s}$ should reflect the uncertainty in the position of the object $i$ at the instant $c_{i j}$ from ${ }_{F} \widehat{\boldsymbol{x}}_{i}^{c_{i j} \mid s}$ and $\widehat{\boldsymbol{C}}_{i i}^{c_{i j} \mid s}-$ i.e. for the obstacle avoidance tasks we have $\nu_{i j}^{s \mid s}=f\left({ }_{F} \widehat{\boldsymbol{x}}_{i}^{c_{i j} \mid s}, \widehat{\boldsymbol{C}}_{i i}^{c_{i j} \mid s}\right)$. At $c_{i j}$, the critical parameters are the predicted object distance $\widehat{d}_{i}$ and its corresponding uncertainty represented by $\widehat{C}_{\widehat{d}_{i}}-$ which can be easily obtained from $\widehat{\boldsymbol{C}}_{i i}^{c_{i j} \mid s}$. With both the predicted distance $\widehat{d}_{i}$ and the statistical deviation $\sigma_{\widehat{d}_{i}}$, the probability $\widehat{P}_{i}^{c_{i j}}$ of collision with the object can be estimated as follows:

$$
P_{i}=\int_{-\infty}^{0} \frac{1}{\sigma_{d_{i}} \sqrt{2 \pi}} e^{\frac{-\left(x-d_{i}\right)^{2}}{2 \sigma_{d_{i}}^{2}}} d x
$$

$P_{i}$ can be calculated either numerically or by means of tables. But in this case, being only interested in the reduction of these values, and not in their evaluation, it may be replaced by $P_{i} \sim \sigma_{d_{i}} / d_{i}$, thus simplifying substantially the calculation efforts.

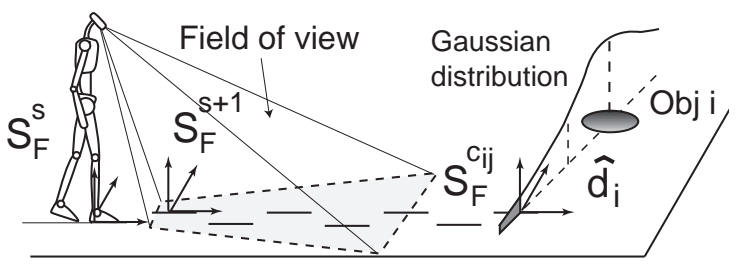

Fig. 3 Definition of the Probability of collision $P_{i}$.

\section{Gaze Control Specific Task Definition}

Given such a situation described by a pre-planned local path, several critical points for collision, the present knowledge about the environment and the characteristics of the walking machine, the task of the gaze control can be defined. The goal consists in minimizing the risk of collision, i.e. the obstacle avoidance Incertitudes $\nu_{i j}$ in order to gather enough information either for passing safely by the object or planning alternative paths around it. This definition holds also in the case of complicated paths, since multiple critical points may appear for each object.

\section{Prediction Algorithm and Gaze Optimization}

The objective is then, as explained in Section IV-A, to define the optimal camera orientation $\widehat{\Omega}_{\star}=\{$ pan, tilt $\}$ that maximizes the predicted acquired $\mathcal{I C}$ in the next measurement process in $s+1$ for the obstacle avoidance task. This can be expressed as a maximum reduction of the predicted Incertitudes, i.e.

$$
\begin{aligned}
\widehat{\boldsymbol{\Omega}}_{\star}^{s+1} & =\arg \max _{\widehat{\boldsymbol{\Omega}}} \sum_{i=1}^{N_{o b j}} \sum_{j=1}^{N_{c_{i}}} \widehat{\mathcal{I C}}_{i j}^{s+1}\left(\widehat{\boldsymbol{\Omega}}, \boldsymbol{x}^{s}, \boldsymbol{C}^{s}, \nu_{i j}^{s+1 \mid s}\right)= \\
& =\arg \max _{\widehat{\boldsymbol{\Omega}}} \sum_{i=1}^{N_{o b j}} \sum_{j=1}^{N_{c_{i}}}\left(\nu_{i j}^{s+1 \mid s}-\hat{\nu}_{i j}^{s+1 \mid s+1}\right)= \\
& =\arg \min _{\widehat{\boldsymbol{\Omega}}} \sum_{i=1}^{N_{o b j}} \sum_{j=1}^{N_{c_{i}}} \hat{\nu}_{i j}^{s+1 \mid s+1}
\end{aligned}
$$

where $N_{o b j}$ is the number of objects present and $N_{c}$ the estimated number of critical points per object. $\widehat{\mathcal{I C}}_{i j}^{s+1}$ is the predicted acquired $\mathcal{I C}$ about the objects' relevance in the critical points $c_{i j}$, and equals the difference $\nu_{i j}^{s+1 \mid s}-\hat{\nu}_{i j}^{s+1 \mid s+1}$. The first term represents the predicted Probability of Collision with the present knowledge, see left column in Fig. 4. The second, this probability if a measurement is taken under the conditions $\widehat{\Omega}$ in the next 
step $s+1$, as indicated in the right column in Fig. 4. Since all the Incertitudes $\nu_{i j}$ represent the same - predicted probabilities of collision, these can be compared directly. All the reductions have the same significance, although some will be reduced more than others, as expected. This is one of the most favorable characteristics of the proposed definition of obstacle avoidance Incertitude in comparison with [3]. Thus, one can assert that the strategy

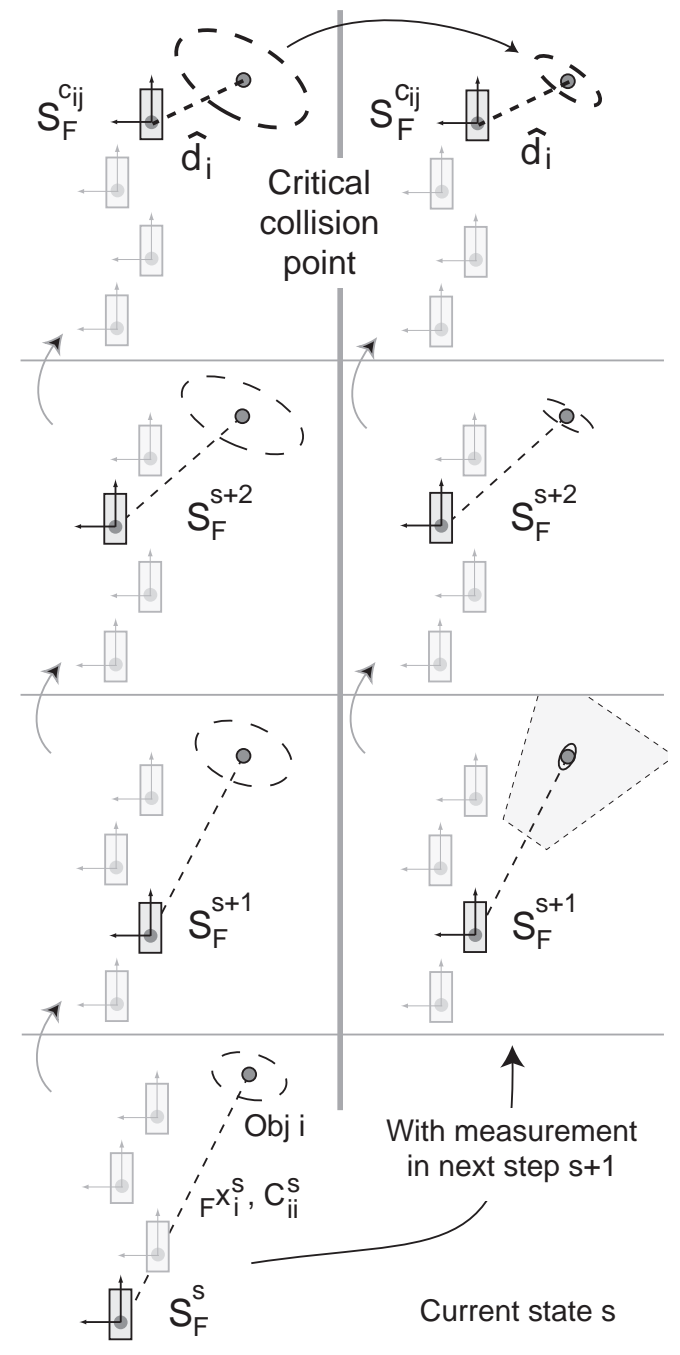

Fig. 4 Definition of obstacle avoidance Incertitute with (right) and without (left) measurement in the next step.

is to behave in such a way that the uncertainty in the objects position at the key instants of time - critical points - is minimized, thus doing at each point in time the best that the accumulated information allows. Future measurements are obviously not taken into account owing to the enormous increase in the alternatives this would imply.

\section{Practical Considerations}

Next, we shall consider some factors involved in the way the information gathering effects the gaze behavior re- sulting from the strategy presented. The specific influence of each of these factors cannot be treated independently, but as an interaction among them. This can be resumed in the influence of four parameters which determine how the $\mathcal{I C}$ varies related to the measurement process.

The first factor is the amount of uncertainty introduced to the state estimation in the walking process until the robot reaches the critical point. This depends on how important the dead-reckoning errors are, cf. Fig. 2. In the case where this uncertainty is considered constant which implies a uniformity in the stepping process - it can be expressed as the number of steps $\left(c_{i j}-s\right)$ left until arriving at object $i$. If a normal step length is assumed for the prediction process, then this measure represents the current relative distance between object and robot. A large distance implies that the information from measuring this object now will be deteriorated to a greater extent till this critical point is actually reached.

Another factor is the predicted critical distance to the object when passing it by $\widehat{d}_{i}$. It is determined by the preplanned local path to be followed. Large distances mean low Probability of Collision, see Fig. 3. The precision in the measurement process [4], and the number of measurements per step are also factors influencing the information gathering strategy. The higher the precision or the greater number of measurements, the higher the gathered amount of information and, therefore, the possible reduction in the uncertainties. The information accumulation increases with high quality perception, meaning that the interest of the object in the next step will be lower.

The last factor is the present amount of information accumulated about object position, represented in $\boldsymbol{C}_{i i}^{s}$. A large present uncertainty implies a high reduction if a measurement takes place, i.e. perception under these conditions is worthy. On the one hand, obviously, the larger is the present uncertainty, the larger the present Incertitude $\nu_{i j}^{s+1 \mid s}$. On the other hand, after a precise measurement at $s+1$, the Incertitude $\hat{\nu}_{i j}^{s+1 \mid s+1}$ will be similar regardless of past information. Thus, the difference $\left(\nu_{i j}^{s+1 \mid s}-\hat{\nu}_{i j}^{s+1 \mid s+1}\right)$ will be greater if the present uncertainty is also high.

\section{IMPLEMENTATION AND SIMULATION}

To test the validity of the proposed approach, simulation experiments were carried out in which a given local path with stochastic deviations in dead-reckoning, was generated for the robot by driving its motion model with Gaussian noises: This simulates a polygonal path composed of straight line segments and the robot's noisy motion over it. The goal of the gaze control is to gather as much path-dependent information about the objects' position as possible. The effects of this goal-oriented perception and the functioning of the information management scheme are described next. 


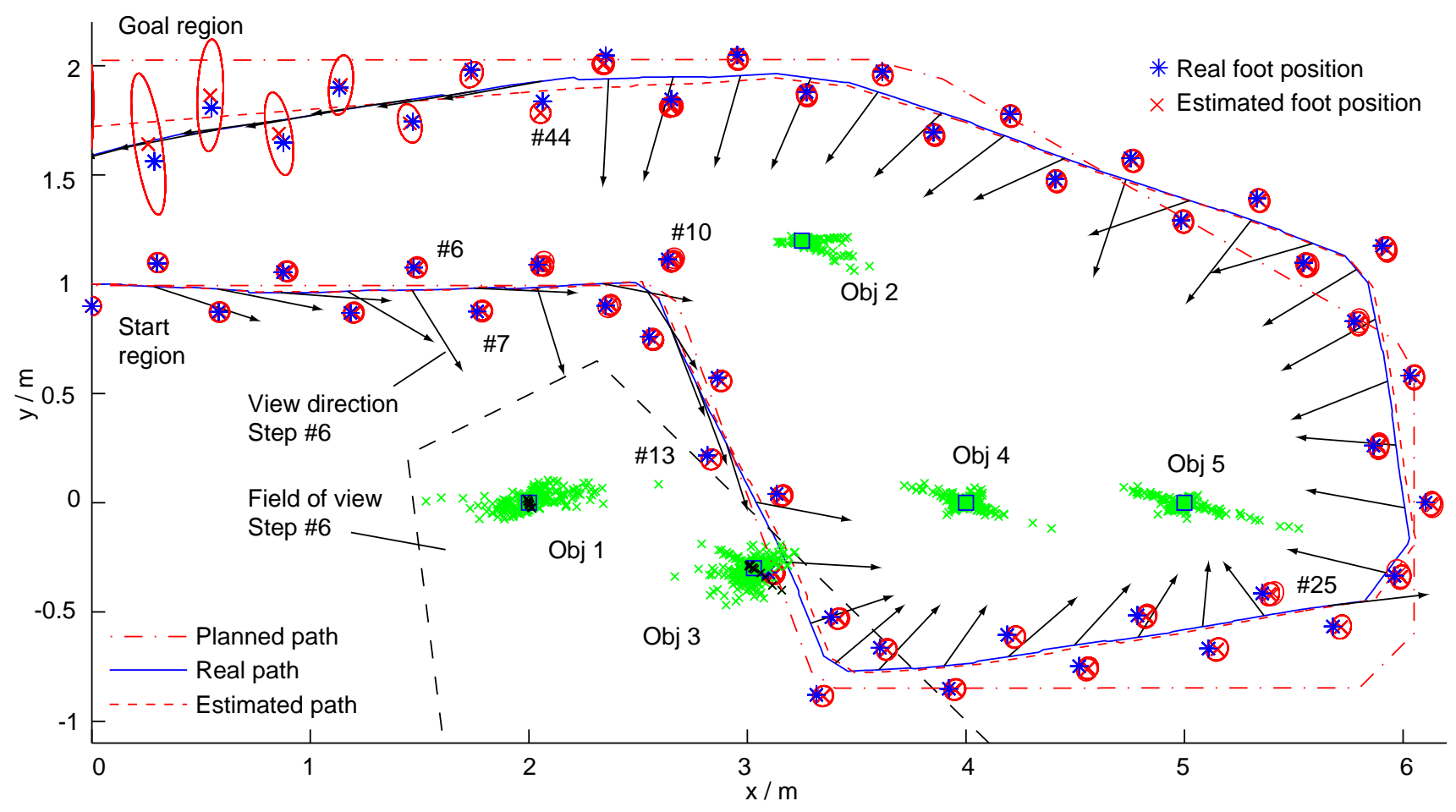

Fig. 5 Simulated scenario with various obstacles, walking motion, measurement process, and intelligent gaze behavior.

\section{A. Simulation Layout}

The robot is commanded to perform position-based navigation along a given path (Planned path from Start region to Goal region, see Fig. 5) as described above, specified by a sequence of step parameters. The robot is driven in a U-shaped scenario. The desired local path has an approximately open rectangular form with side-lengths $6 \times 3 m$ in the vicinity of an irregular distribution of feature points - representing obstacles - of which it is able to make simulated noisy stereo visual measurements. Some of these obstacles, see $\operatorname{Obj} 3$, are deliberately located on the given planned local path and should be avoided - for more details on this topic see [5].

In this simulation, we assume a rather simple walking motion resulting in a continuous constant head movement in a finite 3D-space with objects $(\operatorname{Obj} 1,2,3,4$, and 5 , marked by squares), cf. Fig. 5 . The robot starts walking straight ahead in $x$-direction with constant velocity, and performs steps translating its foot frame $S_{F_{R, L}}$ according to $\left[\begin{array}{llll}x_{S} & \pm y_{S} & 0\end{array}\right]^{T}$ where "+" is used to change from the right to the left foot, and "-" viceversa. Assumed values are $x_{S}=0.3 \mathrm{~m}$ and $y_{S}=0.2 \mathrm{~m}$. The parameter $\theta_{S}$ describing discrete changes in orientation is varied after some steps in order to follow the planned local path. However, robot locomotion causes errors performing steps in both, next foot position and orientation, cf. Fig. 2. In this simulation three sources of white Gaussian noise have been considered: $\sigma_{\Delta x_{S}}=0.005 \mathrm{~m}, \sigma_{\Delta y_{S}}=0.005 \mathrm{~m}$, and $\sigma_{\Delta \theta_{S}}=3^{\circ}$. Errors in the kinematic chain modeling have not been taken into account. The active vision system takes an image of the scenario every $0.02 \mathrm{~m}$ as the robot moves. After a sequence of measurements (14 in this case, marked with crosses), a step is performed, i.e. the foot frame $S_{F_{R, L}}$ changes to the other foot. Assuming the measuring process takes place in camera frame-rate, this corresponds with a walking velocity of $0.6 \mathrm{~m} / \mathrm{s}$, acceptable for current bipeds. For this measurement process a Gaussian nonhomogeneous (foveated) pixel noise with a standard deviation depending on the projecting point has been assumed [6]. The active perception system has only two rotational degrees of freedom: pan and tilt, i.e. $\boldsymbol{\Omega}=\{$ pan, tilt $\}$. The gaze control system makes a prediction once per step and selects then the new view direction.

\section{$B$. Simulation Results}

Fig. 5 shows the results of this simulation. Planned, real, and the estimated robot head motion and feet positions in the world frame are depicted. The operation of the information management scheme is shown by means of the estimated head motion and feet locations - by means of the $90 \%$ confidence ellipses. The resulting gaze behavior is indicated in each step with arrows. For Step \#6, besides the arrow, the field of view on the ground is depicted in order to indicate the range of a measurement. The measurements of $O b j 1$ and $O b j 3$ corresponding to this step are highlighted. The position uncertainties of $O b j 1$ and $\operatorname{Obj} 3$ at this point are shown in detail in Fig. 6 by means of the $90 \%$ confidence ellipsoids (their ground intersection). The latter are calculated 14 times per step 
taking into account the different measurements; they are depicted with crosses. Note the clearly larger first ellipsoids - hard to see in the figures - they are consequence of the errors in the step parameters defined above.

This simulation also demonstrates how the objects are focused with respect to the presence of critical points for collision. For example for $O b j$ 1, among others, steps \#7 and \#13 are critical and Step \#10 for Obj 2. Fig. 5 shows that immediately before reaching these critical points, these objects become more relevant; in further steps, this behavior can be also observed. At the last critical point of the scenario, at Step \#44, no more obstacles are task-relevant, thus the view direction remains in its home position. From this point on, the functioning of the information management scheme can be observed. No extra information is gathered by the perception system anymore and therefore the quality of estimation of the robots position, through objects' perception, is getting worse. At Step \#25 another feature of the gaze controller can be observed. Here the view direction remains in the home position since the physical constraints of the active vision system cannot be violated, as stated in Eq. (3).
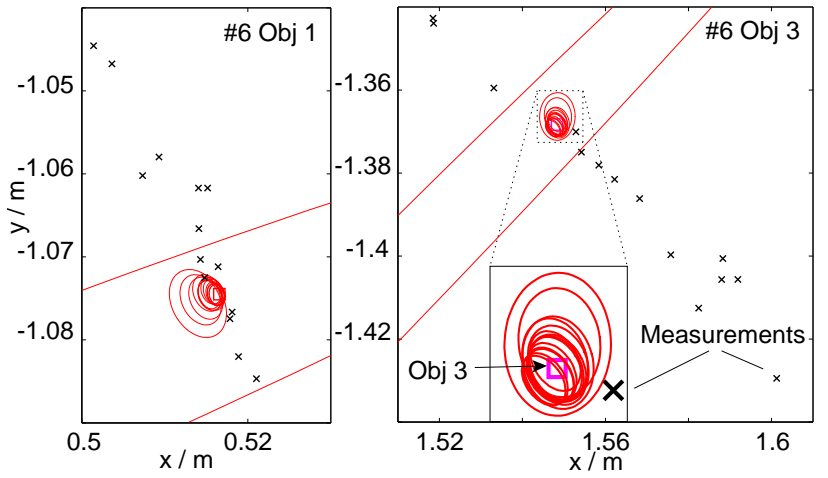

Fig. 6 Reduction of position uncertainty of $O b j 1$ and $O b j 3$ in foot reference frame $S_{F}$ at $S t e p \# 6$.

It is worth noting that the same obstacle can get a very high relevance in two or more different situations, i.e. the robot does not "forget" an obstacle, in case it can become dangerous later on or can still contribute useful information if observed from another perspective. Fixation on only one feature implies that robot angular deviations are hardly estimated and consequently, a correction of the other obstacles position estimations can not be performed.

From a practical point of view some of the procedures described here might not be totally appropriate for implementation on a real robot. Although the optimal behavior would be achieved applying the method presented in a generic formulation, i.e. with an unlimited number of critical points and a large planning horizon, very similar behavior has been achieved by limiting these parameters, with a ten-steps planning horizon in the presented simulation. With a limited planning horizon, the behavior obtained is fairly equivalent to the one presented without limitation in the prediction and the computation time decreases orderly.

\section{CONCLUSIONS AND FUTURE WORK}

In this article we have presented an approach to gaze control integrating active information gathering and local path following requirements for obstacle avoidance. This novel, biologically inspired predictive gaze control strategy for active vision systems rests on two new concepts: Information Content and Incertitude. A redefinition of the Incertitude in the context of vision guided humanoid obstacle avoidance during walking allows the inclusion of the planned local path-dependence. The general strategy is based on the maximization of the predicted visual information. For the information/uncertainty management a coupled hybrid Extended Kalman Filter is employed. The performance resulting from this view direction control scheme shows the dependence of the intelligent gazing behavior on the planned local path.

In order to fulfill the requirements of autonomous locomotion, these proposed conceptions for obstacle avoidance must be combined with other strategies related to concurrent navigational task, e.g. self-localization or exploration. Some work in this direction will be presented elsewhere.

Acknowledgments: This work was supported in part by the German Research Foundation (DFG) within the "Autonomous Walking” Priority Research Program.

\section{REFERENCES}

[1] K. Nishiwaki, S. Kagami, J. J. Kuffner, K. Okada, Y. Kuniyoshi, M. Inaba, and H. Inoue, "Online humanoid locomotion control by using $3 \mathrm{~d}$ vision information," in Procs. of the I8th Int. Symp. on Exp. Robotics, Sant'Angelo d'Ischia, Italy, July 2002. 1

[2] K. Yokoi, F. Kanehiro, K. Kaneko, K. Fujiwara, S. Kajita, and H. Hirukawa, "Experimental study of biped locomotion of humanoid robot HRP-1s," in Procs. of the I8th Int. Symp. on Exp. Robotics, Sant'Angelo d'Ischia, Italy, July 2002. 1

[3] J. F. Seara, K. H. Strobl, and G. Schmidt, "Information management for gaze control in vision guided biped walking," in Procs. of the IEEE/RSJ Int. Conf. on Intelligent Robots and Systems IROS, Lausanne, Switzerland, 2002, pp. 31-36. 1,4

[4] O. Lorch, J. F. Seara, K. H. Strobl, U. D. Hanebeck, and G. Schmidt, "Perception Errors in Vision Guided Walking: Analysis, Modeling, and Filtering," in Procs. of the IEEE Int. Conf. on Robotics and Automation, Washington DC, USA, 2002, pp. 2048-2053. 1, 2, 4

[5] O. Lorch, A. Albert, J. Denk, M. Gerecke, R. Cupec, J. F. Seara, W. Gerth, and G. Schmidt, "Experiments in visionguided biped walking," in Procs. of the IEEE/RSJ Int. Conf. on Intelligent Robots and Systems IROS, Lausanne, Switzerland, 2002, pp. 2484-2490. 2, 5

[6] L. Matthies and S. A. Shafer, "Error modeling in stereo navigation," IEEE Journal of Robotics and Automation, vol. 3, no. 3, June 1987 . 5 